\title{
Model-based Segmentation of Cortical Regions of Interest for Multi-subject Analysis of fMRI Data
}

\author{
Karin Engel ${ }^{1}$, André Brechmann ${ }^{2}$, Klaus Toennies ${ }^{1}$ \\ ${ }^{1}$ Otto-von-Guericke University, Magdeburg, Germany \\ ${ }^{2}$ Leibniz Institute for Neurobiology, Magdeburg, Germany \\ engel@isg.cs. uni-magdeburg.de
}

\begin{abstract}
The high inter-subject variability of human neuroanatomy complicates the analysis of functional imaging data across subjects. We propose a method for the correct segmentation of cortical regions of interest based on the cortical surface. First results on the segmentation of Heschl's gyrus indicate the capability of our approach for correct comparison of functional activations in relation to individual cortical patterns.
\end{abstract}

\section{Introduction}

The current approach in functional brain imaging is to average the data across subjects and map the resulting brain activation on a standard brain (i.e. Talairach or Montreal Neurological Institute (MNI)). A prerequisite for the precise localisation of functional areas, however, is to correctly register any given brain volume to the brain template used as standard space. Different registration methods are used to compensate either only the brain size by linear transformation or the relative location and shape of gross anatomical structures, i.e. prominent sulci and gyri, by non-linear transformations both implemented in fMRI analysis software like SPM (http://www.fil.ion.ucl.ac.uk/spm/) or BrainVoyager (http://brainvoyager.com). Current registration methods, however, do not adequately consider inter-individual differences in configuration of specific brain regions, e.g. the auditory cortex (AC) [3]. The alternative approach is to define subject-specific regions of interest (ROI) by using a combination of anatomical landmarks and clusters of brain activation obtained in functional imaging studies $[1,6]$. So far, the observer-dependent and time-consuming nature of the manual delineation of ROI represents a tradeoff for the advantages (better spatial specificity and improved statistical power) of this approach.

Therefore, we developed a hierarchical Finite Element Model of the auditory cortical folding pattern and applied it to the automatic surface-based labeling of Heschl's gyrus [4]. Here, we use our model for segmentation of the landmarkoriented auditory ROI described in $[1,6]$. This provides a reliable surface-based comparison of auditory functional activations across subjects. 


\section{Method}

We use an algorithm that allows for the sequential segmentation of landmarkrelated cortical ROI based on the cortical surface. Functional activations are analysed and classified at a regional level.

\subsection{Definition and Segmentation of the ROI}

Our algorithm utilises the properties of the cortical surface, represented as flat maps (Fig. 1(a)). This firstly requires the inner cortical surface (white - grey matter boundary) to be extracted based on the exact segmentation of the grey and white matter according to [8]. The triangulated cortical surface of each hemisphere is then processed to create flat maps using BrainVoyager.

The empirical system of landmark-related ROI serves to parcellate the AC into four areas with known differential activation $[1,6]$. The ROI form adjacent territories in the individual hemispheres in relation to the anatomical landmarks depicted in Fig. 1(b). ROI T1 follows the course of Heschl's gyrus (HG) on its anteromedial rim and extends on the lateral aspect of gyrus temporalis superior (lGTS). TA covers planum polare anterior to HG, T2 is centered to Heschl's sulcus (HS), and T3 covers the posterior planum temporale (PT).

A collection of finite element models (FEM) $[7,4]$ represents the structural decomposition of the auditory cortical folding pattern into different gyri and

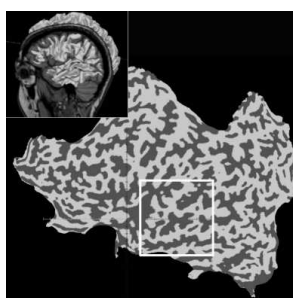

(a)

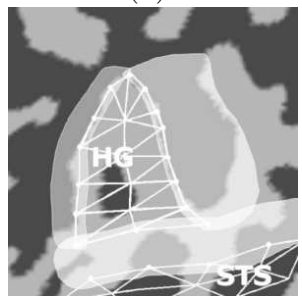

(e)

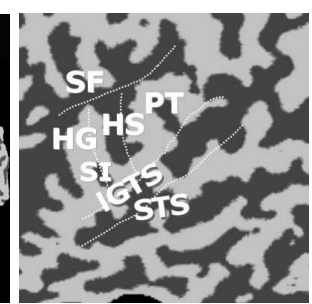

(b)

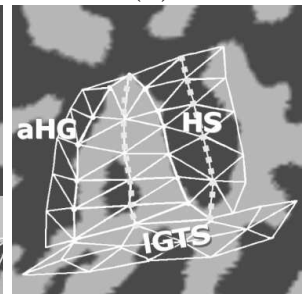

(f)

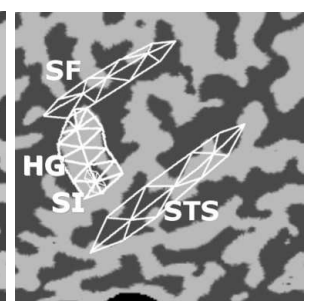

(c)

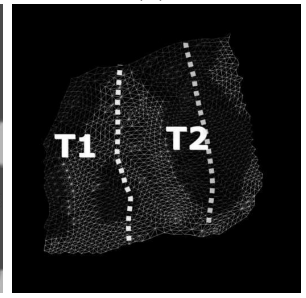

(g)

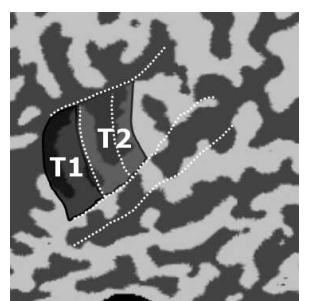

(d)

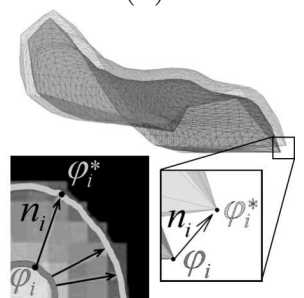

(h)

Fig. 1. Identification of anatomical landmarks and surface-based segmentation of auditory ROI. Our algorithm utilises the properties of 2D flat maps of the cortical surface (a) and detailled (b)-(g). Color indicates gyri (light), sulci (dark) and background (black). Each flat map vertex $\varphi_{i}$ is associated with its position on the $3 \mathrm{D}$ folded cortical surface (h) 
sulci, namely HG - which may or may not show a sulcus intermedius (SI) -, Sylvian Fissure (SF) and sulcus temporalis superior (STS). It represents HG as the central part of the hierarchical FEM of the AC (Fig. 1(c)). Automatic segmentation of the landmarks in the individual flat maps can be accomplished by a combination of the dynamic FEM with an evolutionary strategy, as described in [4], or with user interaction (Sec. 3).

The temporal lobe is then further subdivided. In this work, we are only interested in T1 and T2 (Fig. 1(d)), i.e. labeling of HS, the anteromedial rim of HG (aHG) and lGTS completes our parcellation. The shape models of HG and STS - which are segmented in the previous step of our algorithm - define expectation maps for the adjacent HS, aHG and lGTS (Fig. 1(e)). These are likewise represented as dynamic FEM. The algorithm initialises the FEM based on the parametrisation of the HG and STS model, i.e. it uses the final displacements of the matched shape models' boundary nodes (Fig. 1(f)).

Based on the segmented anatomical landmarks, the temporal lobe of each individual subject can be easily parcellated into the two auditory ROI. Therefore, flat map vertices segmented by the FEM of aHG, HG and HS are combined. Finally, ROI T1 and T2 are separated by the medial axis nodes of the HG model. Each flat map vertex $\varphi_{i}$ is now assigned label $l_{b}$ for background, or contributes to the inner cortical surface of ROI $r \in\{\mathrm{T} 1, \mathrm{~T} 2\}$, i.e. $\varphi_{i}=l_{r}$ (Fig. $1(\mathrm{~g})$ ). The corresponding outer cortical surface of ROI $r$ is represented by vertices $\left\{\varphi_{i}^{*}=l_{r}\right\}$, which are estimated by sampling the pre-segmented grey matter along the surface normals $n_{i}$ (Fig. 1(h)). The voxels enclosed by $\left\{\varphi_{i}=l_{r}\right\}$ and $\left\{\varphi_{i}^{*}=l_{r}\right\}$ constitute the 3D-ROI masks used for analysis of fMRI data.

\subsection{Surface-based ROI Analysis of the fMRI Data}

The activation profiles for all voxels $v_{j}$ within a ROI mask are extracted and modeled using the general linear model [5]. Contrasting with the standard voxellevel analyses, the statistical tests are performed on the regional activations. Activation clusters exceeding a spatial extent of 8 contiguous voxels at the given significance level, e.g. $p<.001$, are considered for further analyses.

In our case, we are interested in comparing the activation patterns within ROI across subjects. The clustered functional volumes are therefore projected

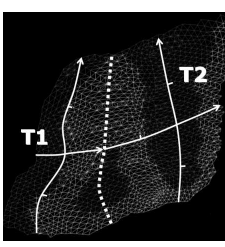

(a)

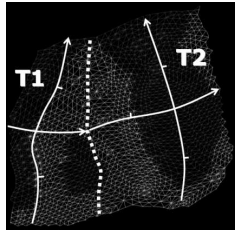

(b)

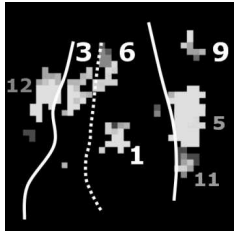

(c)

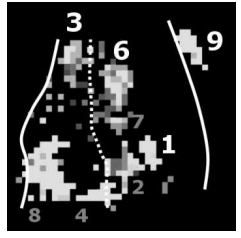

(d)

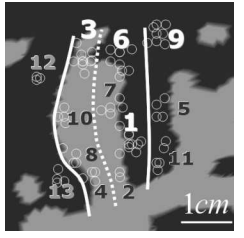

(e)

Fig. 2. Classification of functional activations: auditory ROI T1 and T2 in left hemisphere (LH) for two different subjects (a)-(d); activations are displayed in resolution of voxels $v_{j}(\mathrm{c}),(\mathrm{d})$; activation peaks for nine subjects mapped onto a template (e) 
Table 1. The (mean \pm standard deviation of the) Hausdorff-distance $\delta_{H}$ of the 2D ROI boundaries and the region overlap $\omega$ of the 3D ROI masks were computed for comparison with the expert's segmentations of nine data sets

\begin{tabular}{c|cccc}
\hline ROI & T1, LH & T2, LH & T1, RH & T2, RH \\
\hline$\delta_{H}$ in $m m$ & $0.12 \pm 0.1$ & $0.19 \pm 0.12$ & $0.1 \pm 0.09$ & $0.16 \pm 0.11$ \\
$\omega$ in $\%$ & $95.0 \pm 2.9$ & $94.1 \pm 3.6$ & $94.5 \pm 4.0$ & $94.0 \pm 4.1$ \\
\hline
\end{tabular}

onto the individual cortical surfaces. We employ two different volume-to-surface projection methods which define a mapping $M: \varphi_{i} \mapsto v_{j}$. The first method is to consider each vertex $\varphi_{i}=l_{r}$ of the 3D inner cortical surface of ROI $r$ and assign to it the activation of the voxel with which it intersects $\left(M_{I}\right)$. Second, the activation along the entire grey matter thickness of the ROI is considered. It is defined by pairs of vertices $\left(\varphi_{i}=l_{r}, \varphi_{i}^{*}\right)$ (Fig. 1(h)). The maximum activation along this segment is mapped to $\varphi_{i}\left(M_{T S}\right)$.

A local, object-centered coordinate system is established in each individual data set by the model-based segmentation of the ROI. This allows for the detailed classification of the activations based on the spatial position w.r.t. the coordinates. In our case, activations within $\mathrm{T} 2$ can be differentiated by their relation to the medial axis nodes of the HS model, which constitute the $y$-coordinate (lateral-medial). Linear interpolation of the nodal displacements along the boundaries of the HG and HS models establishes the $x$-coordinate (anterior-posterior) within T2. Equivalent clusters within T1 can be identified based on the $x$-coordinate which is defined similarly based on the boundaries of aHG and HG. The nodes which are shared by the aHG and HG models represent the $y$-coordinate (lateral-medial) within T1 (Figs. 2(a) and 2(b)).

\section{$3 \quad$ Results}

The two auditory ROI in the left and right hemispheres ( $\mathrm{LH}$ and $\mathrm{RH}$ ) were parcellated for nine subjects. A gold standard was given in terms of manual segmentations of the ROI in 2D (on the flat maps) and in the 3D MRI data sets.

\subsection{Quality and Performance of the ROI Mask Segmentation}

In this work, we interactively initialised the prototypical finite element meshes which represent the shape and structural configuration of HG, (SI), SF and STS (Fig. 1(c)). More specifically, instances of the hierarchical FEM of the auditory folds were placed close to the position of the landmark SF in each individual flat map and adequately scaled, i.e. two mouse clicks were required for initialisation. This allows examining the adequacy of our segmentation method based on correctly identified landmarks HG, SF and STS (note, that FEM of HS, aHG and lGTS are initialised automatically). For all data sets segmentation of ROI T1 and $\mathrm{T} 2$ in $2 \mathrm{D}$ stopped after $7-20$ seconds. While manual delineation of the ROI in a 3D MRI data set took a trained expert $45-70$ minutes, the 3D-ROI masks 
were computed in about 30 seconds on a standard PC (3.2GHz P4, Matlab/C). As given in Table 1, our segmentations are comparable with the gold standard in 2D (which proves correct extrapolation of the individual ROI boundaries) and 3D (which shows accuracy in sampling the ROI's grey matter volume).

\subsection{Surface-based fMRI ROI Analysis}

To proof our concept of surface-based labeling of functional activations, we applied our method to the auditory fMRI study of Deike et al. [2]. We used the $M_{T S}$ mapping before labeling of the activation clusters, because we found that at average $67 \%$ more activation in the cortical grey matter was captured compared with the mapping $M_{I}$.

While usually a ROI analysis computes the coordinate of the activation peak in standard space, our method considers any ROI activations and computes several candidates for fields of specific functionality. Moreover, the contribution of individual activations can be analysed w.r.t. the group average ROI activations. In our case, the inter-subject comparison of activation patterns relies on labels which represent relations to anatomical landmarks. Figures 2(c) and 2(d) show multiple labeled clusters of auditory activation for two subjects. Dotted and solid lines represent $\mathrm{HG}$ and the $y$-coordinates of $\mathrm{T} 1$ and $\mathrm{T} 2$, respectively. Figure 2(e) shows activation peaks of identical clusters in all nine subjects mapped to a template based on the displacements of the parametric ROI model in the individual and template cortical surfaces. Clusters with equivalent labels varied of up to $8 \mathrm{~mm}$ in standard space. However, the spatial configuration was consistent among subjects (for example, clusters 1,3,6 and 9 were present in all nine subjects, cluster 12 in three subjects), which suggests functional correspondence.

\section{Discussion}

Using our hierarchical deformable shape model, individual landmark-related ROI can be segmented almost automatically and very precisely. The parametric ROI model supports a detailed functional parcellation of the human neocortex.

\section{References}

1. Brechmann A, Baumgart F, Scheich H. Sound-level-dependent representation of frequency modulations in human auditory cortex: A low noise fMRI study. J Neurophys 2003; 87: 423-33

2. Deike $\mathrm{S}$, et al. Auditory stream segregation relying on timbre involves left auditory cortex. Neuroreport 2004; 15(9): 1511-4

3. Desai R, et al. Volumetric vs. surface-based alignment for localization of auditory cortex activation. Neuroimage 2005; 26: 1019-29

4. Engel K, Brechmann A, Toennies K. A two-level dynamic model for the representation and recognition of cortical folding patterns. Proc IEEE ICIP 2005; 297-300 
5. Friston K, et al. Statistical parametric maps in functional imaging: A general linear approach. Human Brain Mapping 1995; 2: 189-210

6. Gaschler-Markefski B, et al. Definition of human auditory cortex territories based on anatomical landmarks and fMRI activation. In: Dynamics of Speech Production and Perception, IOS-Press 2006; 5-16

7. Sclaroff S, Pentland A. Modal matching for correspondence and recognition. IEEE Trans Pattern Anal Mach Intell 1995; 17(6): 545-61

8. Wasserthal C, et al. Automatic segmentation of the cortical grey and white matter in MRI using a region growing approach based on anatomical knowledge. Proc BVM 2008; 